\title{
Development of Chemical Probes for the Bromodomains of BRD7 and BRD9
}

Peter G. K. Clark, ${ }^{1,3}$ Darren J. Dixon ${ }^{1}$ and Paul E. Brennan ${ }^{2}$

${ }^{1}$ Department of Chemistry, University of Oxford, Oxford OX1 3TA, UK

2 Structural Genomics Consortium, Target Discovery Institute, and Alzheimer's Research UK Oxford Drug Discovery Institute, Nuffield Dept of Medicine, Oxford OX3 7FZ, UK

Corresponding author: Brennan, P. E. (paul.brennan@sgc.ox.ac.uk)

${ }^{3}$ Present address: Department of Chemistry, Simon Fraser University, Burnaby V5A 1S6, Canada

Keywords: Bromodomain; probe; epigenetic; BRD7; BRD9; structure-based-drug-discovery

Teaser: Small molecule probes have now been developed for exploration of the role and therapeutic potential of the BRD7 and BRD9 bromodomain epigenetic readers. 


\section{Abstract}

The bromodomain family of proteins are "readers" of acetylated lysines of histones, a key mark in the epigenetic code of gene regulation. Without high quality chemical probes with which to study these proteins, their biological function, and potential use in therapeutics, remains unknown. Recently, a number of chemical ligands were reported for the previously unprobed bromodomain proteins BRD7 and BRD9. Herein the development and characterisation of probes against these proteins is detailed, including the preliminary biological activity of BRD7 and BRD9 assessed using these probes. Future studies utilising these chemically-diverse compounds in parallel will allow for a confident assessment of the role of BRD7/9, and give multiple entry points into any subsequent pharmaceutical programs.

\section{Introduction}

Post-translational modification of chromatin represents a constituent part of the epigenetic code. Acetylated lysine residues (Kac) of histones are selectively recognised by a conserved protein interaction module known as a bromodomain (BRD) [1,2]. BRD-containing proteins subsequently induce changes to gene expression through catalytic domains or through anchoring other proteins to the chromatin [3]. The BRD-proteins BRD7 and BRD9 have been implicated in the regulation of gene expression [4] and control of the cell cycle [5-8], in addition to showing an empirical correlation with numerous cancers [8-15]. However, to decipher their biological function and to stimulate the discovery of new potential therapeutics requires the use of chemical probes - small molecules with high affinity and selectivity for the target in vitro, a known mechanism of action and proven cellular activity $[16,17]$. Herein, we summarise the development and application of chemical probes against BRD7 or BRD9.

\section{BRD7 and BRD9 Chemical Probes}

\section{BRD7/9 architecture}

The BRD-binding pocket of BRD7/9 offers a number of opportunities for selective inhibition over other BRDs. The BRD motif comprises four alpha-helixes joined by flexible loops, with the Kac binding site located in one end of the bundle between the ZA and BC-loops [18]. Native Kac marks of histones are recognised through hydrogen-bonds (H-bonds) between the acetyl carbonyl of the Kac and both the terminal $\mathrm{NH}_{2}$ of a conserved asparagine residue and the hydroxyl of a tyrosine, the latter mediated through a constituent water molecule [19]; in BRD9, these residues are N216 and Y173. When compared to BRD4(1), a key selectivity off-target, it can be observed that the lipophilic WPF shelf characteristic of BRDs is inaccessible in BRD9 due to residue changes in the access channel. There are also significant differences in the ZA channel, which, in conjunction with numerous potential H-bond partners in the binding site, presents opportunities for the development of potent, selective ligands. Although no crystal structure of BRD7 has been reported to date, a solution structure has been determined by NMR [20], and the high sequence homology with BRD9 allows the same observations to be reasonably applied.

\section{I-BRD9}

A cross-screen performed at GlaxoSmithKline identified a lead molecule against BRD9. Screening their internal library of compounds for BRD9 activity identified thienopyridone $\mathbf{1}$ with a $\mathrm{pIC}_{50}$ of 6.7 
against BRD9 (measured by Time-resolved Förster resonance energy transfer (TR-FRET, [21]), but only 4.7 against BRD4(1) (Box 1A) [22]. A co-crystal xray structure of BRD9 with compound 1 was solved to identify the binding mode (PDB ID 4UIT). Pyridones are known structural analogues of Kac [23], with the lactam carbonyl of 1 forming the distinctive H-bonds to both N216 and Y173, mediated through a conserved water molecule. Additionally, the dimethoxyphenyl substituent was observed to H-bond with a network of binding site water molecules, whilst the terminal sulfonamide H-bonded to the backbone NH of R217.

An ensuing structure-based drug design program led to the development of I-BRD9 as a BRD9 probe. As the amide carbonyl was believed to drive selectivity over BRD4(1), this position was derivatised to optimise selectivity. Through Pinnick oxidations of aldehydes and subsequent HATU-couplings, a range of amides were obtained; although the importance of the terminal sulfonamide for potency was confirmed, no significant improvements in both potency and selectivity were obtained. Replacement of the amide for a H-bond donor functional group was envisaged to form a new H-bond to I169.

Negishi cyanations followed by Pinner reactions yielded substituted amidines that displayed increased affinity for $\mathrm{BRD} 9$ ( $\mathrm{pIC}_{50} 8.1$ ). Optimisation of the exocyclic phenyl ring to a 3-CF $\mathrm{CF}_{3}$ substituent led to a small decrease in affinity but a marked increase in selectivity for BRD9 over BRD4(1) (160x selectivity compared to 50x). Finally, extension of the $N$-methyl chain of the pyridone to an ethyl substituent yielded I-BRD9, which displayed increased selectivity across other BRDs compared to the methylated parent.

I-BRD9 was characterised and found to possess all the properties of a BRD9 chemical probe. A BROMOscan assay [24] revealed a $\mathrm{pK}_{\mathrm{d}}$ of 8.7 for I-BRD9 against BRD9, whilst profiling against 33 other BRDs showed a minimum of 70x selectivity over all tested BRDs, including 200x selectivity over the closely-related BRD7 (Box 1B). A co-crystal structure of I-BRD9 with BRD9 revealed the same interactions made by lead compound 1: the thienopyridone acted as a Kac mimic with H-bonds to N216 and Y173 through a water molecule, in addition to H-bonds between the amidine and I169, and between the sulfone and R217 (Box 1C). Activity against the native protein was confirmed using a chemoproteomic competition binding assay, with a $\mathrm{pIC}_{50}$ of 7.1 measured. Cellular-activity was also demonstrated using a NanoBRET assay [25] to assess disruption of BRD9-chromatin binding in cells, with a $\mathrm{pIC}_{50}$ of 6.8 for the BRD9-histone $\mathrm{H} 3.3$ interaction observed. I-BRD9 was further profiled to evaluate the selectivity of the compound over a broad range of pharmacological targets, including receptors, transporters, ion channels, kinases and other enzymes, for which no activity at concentrations less than $5 \mu \mathrm{M}$ was observed.

\section{LP99}

A fragment hit against BRD9 was identified during the development of ligands against the BRDs of PCAF and ATAD2 [26,27]. From an intial fragment library screen, the quinolone $\mathbf{2}$ was demonstrated to mimic Kac in the binding site of the BRD protein ATAD2 (Box 1D), which belongs to the same subfamily as BRD7/9. From a co-crystal structure (PDB ID 4QST), H-bonds between the quinolone carbonyl and both N1064 and a conserved water molecule were observed. When the affinity of this compound for BRD9 was assessed, a $\mathrm{pK}_{\mathrm{d}}$ of 5.3 was observed (measured by isothermal titration calorimetry (ITC), [28]). Overlaying BRD9 to this co-crystal structure revealed a shallow hydrophobic cleft at the $\mathrm{C}-4$ position and a large hydrophobic cavity adjacent to the $\mathrm{C}-7$ position that could be targeted for selective binding to BRD9.

A structure-based optimisation program originating from compound $\mathbf{2}$ led to the development of a BRD7/9 probe. Initially, addition of a methyl group at the C-4 position was found to benefit by hydrophobic-interactions with the small hydrophobic cleft [29]. Next, a range of sp $^{3}$-rich heterocycles, which would allow for the incorporation of chirality to effectively fill the three-dimentional space of 
the binding pocket [30], were attached to the C-7 position using a Buchwald-Hartwig coupling and the equivalent bromoquinolone. A $\delta$-lactam was identified, by both differential scanning calorimetry (DSF, [31]) and ITC $\left(\mathrm{pK}_{\mathrm{d}} 6.2\right)$, as the optimal scaffold to exploit the hydrophobic cavity. A co-crystal structure with BRD9 confirmed the same binding mode of the quinolone core as for ATAD2, with the new ring occupying the large hydrophobic cavity, and the lactam carbonyl positioned near to Y106 (PDB ID 4Z6H). A range of substituted $\delta$-lactams were obtained through a nitro-

Mannich/lactamisation cascade for rapid SAR determination [32]: A C-5 NHBoc and a C-6 phenyl group on the lactam were found to be optimal for BRD9 affinity. A co-crystal structure revealed the phenyl ring occupied another hydrophobic groove and new $\mathrm{H}$-bonds were formed between the lactam carbonyl and Y222, and between the NHBoc group and G159 (PDB ID 4Z6I). Optimisation of the phenyl ring identified a para-chloro as giving the greastest selectivity, whilst optimisation of the NHBoc to an isobutyl sulfonamide group was ideal for BRD9 affinity. The enantiomers were resolved and screened, and LP99 was identified as the active enantiomer. An enantioenriched synthesis of LP99 was then achieved starting from an asymmetric nitro-Mannich reaction using cinchona alkaloidderived phase transfer catalysts [33].

Analysis of LP99 resulted in a confirmation of utility as a dual BRD7/9 chemical probe. LP99 was found to have a $\mathrm{pK}_{\mathrm{d}}$ of 7.0 against BRD9 and a lower $\mathrm{pK}_{\mathrm{d}}$ of 6.0 against BRD7 (ITC). Binding to BRD9 was driven by enthalpy $\left(\Delta \mathrm{H}-10.7 \mathrm{kcal} \mathrm{mol}^{-1}\right)$ with a net loss in entropy observed (T $\Delta \mathrm{S}-1.32$ $\mathrm{kcal} \mathrm{mol}^{-1}$ ). To assess the selectivity of LP99, a DSF screen against the 48 expressible BRDs revealved no significant stabilisation other than for BRD7 and BRD9 (Box 1E). A co-crystal structure of LP99 with BRD9, in addition to confirming the absolute stereochemistry, revealed all the binding elements as previously described (Box 1F): the conserved H-bonds of Kac recognition between the quinolone and N216/Y173; an H-bond between the lactone carbonyl and Y222; and an H-bond between the sulfonamide NH and G159. Cellular activity of LP99 was evidenced through a fluorescence recovery after photobleaching assay (FRAP, [34]), where inhibition of BRD9-chromatin interactions were observed at $0.8 \mu \mathrm{M}$. This cellular activity was further confirmed by NanoBRET assays between BRD7 and BRD9 with histones $\mathrm{H} 3.3$ and $\mathrm{H} 4.4$ (pIC $\mathrm{p}_{50}$ 5.2-5.5). As a point of difference from the achiral probe I-BRD9, the importance of chirality to LP99 was evidenced through the alternate enantiomer having no measureable activity against BRD9, readily providing a negative control for use in bioassays.

\section{Indolizine 3}

A lead for BRD9 was obtained as part of the development of a probe for alternative BRDs. GSK2801 is an indolizine-based chemical probe for the BAZ2A and BAZ2B BRDs (Box 1G) [35]. Activity for BRD9 during development prompted the design of a negative control compound that lacked activity for the desired targets: GSK8573 had no activity for BAZ2A/B but significant affinity for BRD9 pK $_{\mathrm{d}}$ 6.0 , ITC). Reviewing the SAR available, replacement of the methoxyphenyl ring with pyridine was observed to convey a high-affinity for BRD9, and spurred investigation into the development of a probe compound.

Guided by biophysical assays, a new BRD7/9 inhibitor was developed. Through a two-step synthesis consisting of quaternisation of substituted pyridines followed by base-promoted [3+2] cycloaddition reactions with substituted alkynes, a range of substituted indolizines were obtained for screening [36]. Initially, replacement of the propoxy substituent for a morpholine residue boosted affinity for BRD9 without increasing affinity for other BRDs. Simple substitutions of the pyridine ring were detrimental to BRD9 binding, however, extension to an imidazopyridine increased affinity. Finally, extension of the alkyl chain of the ketone, or even replacement with a trifluorinated analogue, was found to be 
poorly tolerated. Of the compounds screened, indolizine 3 was identifed as the best ligand against BRD9 and BRD7 for potency whilst minimising off-target binding.

Characterisation of indolizine 3 revealed fulfilment of the requirements for a dual BRD7/9 chemical probe. A high affinity for BRD9 was observed by both an AlphaScreen assay $\left(\mathrm{pIC}_{50} 6.9\right.$, [37]) and ITC $\left(\mathrm{pK}_{\mathrm{d}} 7.2\right)$, a five-fold stronger affinity than for BRD7 ( $\mathrm{pK}_{\mathrm{d}} 6.4$, ITC). The thermodynamics of the binding to these two proteins revealed binding to BRD9 was largely enthalpically driven $(\Delta \mathrm{H}-10.7$ $\left.\mathrm{kcal} \mathrm{mol}^{-1}, \mathrm{~T} \Delta \mathrm{S}-1.32 \mathrm{kcal} \mathrm{mol}^{-1}\right)$ whereas entropic contributions dominated with BRD7 $(\Delta \mathrm{H}-2.64$ $\mathrm{kcal} \mathrm{mol}{ }^{-1}, \mathrm{~T} \Delta \mathrm{S} 5.99 \mathrm{kcal} \mathrm{mol}^{-1}$ ). Wider selectivity of the compound was assessed by a DSF assay, where only limited activity against other BRDs was observed [Box $1 \mathrm{H}]$. To identify the binding mode and confirm the mechanism of action, a BRD9/indolizine 3 co-crystal structure was solved [Box 1I]: The ketone moiety was identified as the Kac mimic, with H-bonds to N216 and Y173 (via a water molecule). Additionally, the indolizine scaffold shared a $\pi-\pi$ interaction with Y222. Cellular activity of compound $\mathbf{3}$ was confirmed by a FRAP assay, with dose-dependent inhibition of interaction with chromatin observed at sub- $\mu \mathrm{M}$ concentrations.

\section{BI-7273 \& BI-9564}

Two further potent BRD7/BRD9 ligands have been recently reported with limited off-target BRD activity. Through parallel screening of fragment libraries by biophysical assays and compound libraries by docking studies, a dimethylpyridinone-based lead was identified [38]; a structure-based drug discovery program originating from this lead led to the development of the napthyridinone-based ligands BI-7273 and BI-9564. BI-7273 showed a strong affinity for both $\mathrm{BRD} 7\left(\mathrm{pIC}_{50} 6.9\right.$, AlphaScreen; $\mathrm{pK}_{\mathrm{d}}$ 9.5, BROMOscan) and BRD9 ( $\mathrm{pIC}_{50}$ 7.7, AlphaScreen; $\mathrm{pK}_{\mathrm{d}}$ 7.8, ITC; 9.1, BROMOscan), whereas BI-9564 showed improved selectivity for BRD9 ( $\mathrm{pIC}_{50}$ 7.1, AlphaScreen; $\mathrm{pK}_{\mathrm{d}}$ 7.9, ITC; 8.2, BROMOscan) over BRD7 ( $\mathrm{pIC}_{50}$ 5.5, AlphaScreen; $\mathrm{pK}_{\mathrm{d}} 6.6$, ITC), 7.1 (BROMOscan)). Cellular activity against these targets was confirmed through a FRAP assay, with both compounds showing complete inhibition of BRD7/9 association with chromatin at or below 1 $\mu \mathrm{M}$. Significant in vitro activity against the BRD protein CECR2 was observed with both BI-7273 $\left(\mathrm{pK}_{\mathrm{d}}\right.$ 6.7, ITC; 8.1, BROMOscan) and BI-9564 (pK $\mathrm{p}_{\mathrm{d}} 6.7$, ITC; 7.1, BROMOscan); the latter, however, showed no activity in a cellular CECR2 FRAP assay at $1 \mu \mathrm{M}$. This affinity aside, both compounds showed no activity amongst other BRDs at $10 \mu \mathrm{M}$ in a number of assays. Further profiling of selectivity against a wider range of biological targets showed minimal activity against a range of kinases and G-protein coupled receptors. Kac recognition is mediated through the napthyridinone, with the carbonyl oxygen replicating the key H-bonds to N216 and Y173 (PDB ID $5 \mathrm{~F} 1 \mathrm{H})$, in a similar mode to the binding of I-BRD9 and LP99.

\section{Other BRD7/9 ligands}

A number of other ligands for BRD7/9 have been described, however affinity for other BRDs, especially the BET family of proteins, is common. The 1,2,4-triazole bromosporine has been reported by the SGC as a pan-BRD ligand, with activity against atleast 14 BRDs documented by DSF, including BRD9 $\left(\Delta \mathrm{T}_{\mathrm{m}} 3.9^{\circ} \mathrm{C}\right.$, http://www.thesgc.org/chemical-probes/Bromosporine). A platform discovery approach targeting the 1,2,4-triazole motif led to the development of compound 4 [39]. This ligand demonstrated a high affinity for $\mathrm{BRD} 9\left(\mathrm{pIC}_{50} 6.7\right.$, AlphaScreen) in addition to other structurally-unrelated BRDs. A co-crystal structure with BRD9 demonstrated the adjacent triazole nitrogen atoms forming the conserved H-bonds of Kac recognition. Alternatively, a structure-based drug discovery program based around a purine as a Kac mimic resulted in the development of compound 5, which had a high affinity for BRD9 $\left(\mathrm{pK}_{\mathrm{d}} 6.6\right.$, ITC) and also the BET family of proteins 
$\left(\mathrm{pK}_{\mathrm{d}}\right.$ 5.7-5.9) [40]. A co-crystal structure with BRD9 could not be obtained to confirm the binding mode, however cellular activity of this compound against BRD9 was confirmed with a NanoBRET assay ( $\mathrm{pIC}_{50}$ of 6.3). Finally, a patent from Constellation Pharmaceuticals and Genentech claims the use of BRD7/9 inhibitors in Th2-mediated inflammatory disease [41]. The patent claims at least one selective compound exists with some supporting data, however the structure was not disclosed and the compounds that are displayed are from an earlier patent detailing potent BET inhibitors [42].

\section{Use of probes to study BRD7 and BRD9 function}

BRD7/9 probes have only shown limited application to date, however, some promising results have been identified. The effect of I-BRD9 compared to the BET inhibitor I-BET151 on gene expression was assessed in Kasumi-1 cells [22]. The microarray identified a total of 700 genes differentially upor down-regulated by I-BRD9, with subsequent confirmation by qPCR identifying four genes involved in immunological and cancer pathways that were downregulated by I-BRD9 (Figure 2A). Support for the role of BRD7/9 in inflammatory pathways was provided through the use of LP99. The expression of pro-inflammatory cytokines by a human THP-1 monocytic cell line stimulated with lipopolysaccharide was assessed by an ELISA assay [29]; secretion of interleukin-6, a key proinflammatory cytokine, was found to be inhibited in a dose-dependent manner by LP99 (Figure 2B). These two studies represent the first demonstration of a role for BRD7/9 in the regulation of inflammation, potentially through their role in the major chromatin remodelling SWI/SNF complex [43]. These preliminary studies highlight the potential to develop novel anti-inflammatory treatments based on BRD7/9 inhibition.

The implication in cancer pathways was confirmed with evidence of efficacy in a murine leukemia study. BI-7273 and BI-9564 both demonstrated antiproliferative effects against a panel of cancer cell lines: $\mathrm{EC}_{50} \mathrm{~S}$ of $1.4 \mu \mathrm{M}$ and $0.8 \mu \mathrm{M}$, respectively, were measured against the most susceptible cell line, human acute myeloid eosinophilic leukemia cell line EOL-1 [38]. Further investigation revealed that BI-7273 resulted in partial inhibition of MYC expression in these cells. BRD9 was confirmed as the biological target through a domain-swap experiment, wherein the BRD of BRD9 was replaced with that of BRD4, resulting in a loss of antiproliferative activity. The pharmacokinetic profiles of the compounds were then assessed and found to be conducive to in vivo studies. Treatment of BI-9564 in a disseminated mouse model of human acute myeloid eosinophilic leukemia resulted in a median tumor growth inhibition of 52\%. This treatment also resulted in a small improvement in the median survival of the animals, which increased from 20 days to 22 days. Although the effects of treatment were modest, this confirmed targeting BRD7/9 as a means to modulate some forms of cancer, in addition to confirming the use of BI-9564 in in vivo studies of BRD7/9.

\section{Discussion}

In target discovery, the use of high quality chemical probes is pivotal to the correct validation, or invalidation, of pharmaceutically-relevant biomolecules. Probes that selectively modulate one function of often multifunctional proteins are important, as the phenotype of inhibiting all functions, through knock-downs or genetic mutants, does not always correlate with that of inhibiting the one [44]. Further, the development of a single probe is insufficient as it is impossible to interrogate, from the biological data, false positives from off-target effects or false negatives from a deficiency in the probe [45]. The development of numerous probes with different chemotypes is of high importance, as the off-target affinity of one chemotype is unlikely to be reproduced with other motifs. Towards this, I-BRD9, LP99 and indolizine 3 can all be seen to feature different chemical scaffolds that individually complement the binding site of BRD9 but do not significantly overlap amongst themselves (Figure 3). Additionally, the presence of different architectures presents multiple entry points into any subsequent 
pharmaceutical development process. The results obtained to date through the use of a single probe should be confirmed with the other probes now available, to confirm the effects on gene expression, inflammation and the proliferation of cancer cell lines are indeed a result of modulation of BRD7 and BRD9. Further, all available probes should be used concurrently in future investigations into the activities of BRD7/9 to ensure the reliability of the data. Towards this, inactive probes, such as the opposite enatiomer of LP99, should also be incorporated in these studies as control compounds to add confidence to any resulting conclusions.

\section{Conclusions}

Through a number of discovery programs originating from different leads, a range of chemical probes for the selective inhibition of BRD9 (I-BRD9) or BRD7 and BRD9 (LP99, indolizine 3, BI-7273, BI9564) have been developed. These probes have all been confirmed to be potent, selective, active in cells and had their mechanism confirmed through co-crystal structures. Initial testing has implicated BRD7/9 in the regulation of inflammatory and cancer-related genes, in cytokine expression and in the modulation of some forms of cancer. Future experimentation with these probes in parallel will reliably expose the roles of BRD7/9, and give multiple entry points to any ensuing drug discovery program.

\section{Acknowledgements}

P. G. K. C. gratefully acknowledges the Woolf Fisher Trust, the CREATE ChemNET programme and the Centre for Drug Research and Development through a grant awarded to Prof Robert Young. The SGC is a registered charity (number 1097737) that receives funds from AbbVie, Bayer Pharma AG, Boehringer Ingelheim, Canada Foundation for Innovation, Eshelman Institute for Innovation, Genome Canada, Innovative Medicines Initiative (EU/EFPIA) [ULTRA-DD grant no. 115766], Janssen, Merck \& Co., Novartis Pharma AG, Ontario Ministry of Economic Development and Innovation, Pfizer, São Paulo Research Foundation-FAPESP, Takeda, and Wellcome Trust [092809/Z/10/Z].

\section{Conflict of Interest}

The authors declare no competing interests.

\section{References}

[1] Haynes SR, Dollard C, Winston F, Beck S, Trowsdale J, Dawid IB. The bromodomain: a conserved sequence found in human, Drosophila and yeast proteins. Nucleic Acids Res $1992 ; 20 ; 2603$.

[2] Filippakopoulos P, Picaud S, Mangos M, Keates T, Lambert JP, Barsyte-Lovejoy D, et al. Histone recognition and large-scale structural analysis of the human bromodomain family. Cell 2012;149:214-31.

[3] Filippakopoulos P, Knapp S. Targeting bromodomains: epigenetic readers of lysine acetylation. Nat Rev Drug Discov 2014;13:337-56.

[4] Kadoch C, Hargreaves DC, Hodges C, Elias L, Ho L, Ranish J, et al. Proteomic and bioinformatic analysis of mammalian SWI/SNF complexes identifies extensive roles in human malignancy. Nat Genet 2013;45:592-601.

[5] Burrows AE, Smogorzewska A, Elledge SJ. Polybromo-associated BRG1-associated factor components BRD7 and BAF180 are critical regulators of $\mathrm{p} 53$ required for induction of 
replicative senescence. Proc Natl Acad Sci USA 2010;107:14280-5.

[6] Zhou J, Ma J, Zhang BC, Li XL, Shen SR, Zhu SG, et al. BRD7, a novel bromodomain gene, inhibits G1-S progression by transcriptionally regulating some important molecules involved in ras/MEK/ERK and Rb/E2F pathways. J Cell Physiol 2004;200:89-98.

[7] Peng C, Liu HY, Zhou M, Zhang LM, Li XL, Shen SR, et al. BRD7 suppresses the growth of nasopharyngeal carcinoma cells (HNE1) through negatively regulating b-catenin and ERK pathways. Mol Cell Biochem 2007;303:141-9.

[8] Drost J, Mantovani F, Tocco F, Elkon R, Comel A, Holstege H, et al. BRD7 is a candidate tumour suppressor gene required for p53 function. Nat Cell Biol 2010;12:380-9.

[9] Huang H-T, Chen S-M, Pan L-B, Yao J, Ma H-T. Loss of function of SWI/SNF chromatin remodeling genes leads to genome instability of human lung cancer. Oncol Rep 2015;33:28391.

[10] Atkin NB. Significance of chromosome 5 and 17 changes in the development of carcinoma of the cervix uteri. Cytogenet Cell Genet 2000;91:44-6.

[11] Scotto L, Narayan G, Nandula SV, Subramaniyam S, Kaufmann AM, Wright JD, et al. Integrative genomics analysis of chromosome $5 \mathrm{p}$ gain in cervical cancer reveals target overexpressed genes, including Drosha. Mol Cancer 2008;7:58-67.

[12] Kang JU, Koo SH, Kwon KC, Park JW, Kim JM. Gain at chromosomal region 5p15.33, containing TERT, is the most frequent genetic event in early stages of non-small cell lung cancer. Cancer Genet Cytogenet 2008;182:1-11.

[13] Cleary SP, Jeck WR, Zhao X, Chen K, Selitsky SR, Savich GL, et al. Identification of driver genes in hepatocellular carcinoma by exome sequencing. Hepatology 2013;58:1693-702.

[14] Wu W-J, Hu K-S, Chen D-L, Zeng Z-L, Luo H-Y, Wang F, et al. Prognostic relevance of BRD7 expression in colorectal carcinoma. Eur J Clin Invest 2013;43:131-40.

[15] Park YA, Lee JW, Kim HS, Lee YY, Kim TJ, Choi CH, et al. Tumor suppressive effects of Bromodomain-containing protein 7 (BRD7) in epithelial ovarian carcinoma. Clin Cancer Res 2014;20:565-75.

[16] Frye S V. The art of the chemical probe. Nat Chem Biol 2010;6:159-61.

[17] Arrowsmith CH, Audia JE, Austin C, Baell J, Bennett J, Blagg J, et al. The promise and peril of chemical probes. Nat Chem Biol 2015;11:536-41.

[18] Mujtaba S, Zeng L, Zhou M-M. Structure and acetyl-lysine recognition of the bromodomain. Oncogene 2007;26:5521-7.

[19] Owen DJ, Ornaghi P, Yang JC, Lowe N, Evans PR, Ballario P, et al. The structural basis for the recognition of acetylated histone $\mathrm{H} 4$ by the bromodomain of histone acetyltransferase gcn5p. EMBO J 2000;19:6141-9.

[20] Sun H, Liu J, Zhang J, Shen W, Huang H, Xu C, et al. Solution structure of BRD7 bromodomain and its interaction with acetylated peptides from histone $\mathrm{H} 3$ and $\mathrm{H} 4$. Biochem Biophys Res Commun 2007;358;435-441.

[21] Moshinsky DJ, Ruslim L, Blake RA, Tang F. A Widely Applicable, High-Throughput TRFRET Assay VEGFR-2 as a Prototype. J Biomol Screen 2003;8:447-52.

[22] Theodoulou NH, Bamborough P, Bannister AJ, Becher I, Bit RA, Che KH, et al. Discovery of I-BRD9, a Selective Cell Active Chemical Probe for Bromodomain Containing Protein 9 Inhibition. J Med Chem 2016;59:1425-1439.

[23] Vidler LR, Filippakopoulos P, Fedorov O, Picaud S, Martin S, Tomsett M, et al. Discovery of novel small-molecule inhibitors of BRD4 using structure-based virtual screening. J Med Chem 2013;56:8073-88.

[24] Quinn E, Wodicka L, Ciceri P, Pallares G, Pickle E, Torrey A, et al. Abstract 4238, Cancer Res 
2013;73:4238.

[25] Machleidt T, Woodroofe CC, Schwinn MK, Méndez J, Robers MB, Zimmerman K, et al. NanoBRET - a Novel BRET Platform for the Analysis of Protein-Protein Interactions. ACS Chem Biol 2015;10:1797-804.

[26] Chaikuad A, Petros AM, Fedorov O, Xu J, Knapp S. Structure-based approaches towards identification of fragments for the low-druggability ATAD2 bromodomain. Medchemcomm 2014;5:1843-8.

[27] Chaikuad A, Lang S, Brennan PE, Temperini C, Fedorov O, Hollander J, et al. Structure-Based Identification of Inhibitory Fragments Targeting the p300/CBP-Associated Factor Bromodomain. J Med Chem 2016;59:1648-1653.

[28] Ladbury JE, Chowdhry BZ. Sensing the heat: the application of isothermal titration calorimetry to thermodynamic studies of biomolecular interactions. Chem Biol 1996;3:791-801.

[29] Clark PGK, Vieira LCC, Tallant C, Fedorov O, Singleton DC, Rogers CM, et al. LP99: Discovery and Synthesis of the First Selective BRD7/9 Bromodomain Inhibitor. Angew Chem Int Ed 2015;54:6217-21.

[30] Lovering F, Bikker J, Humblet C. Escape from Flatland: Increasing Saturation as an Approach to Improving Clinical Success. J Med Chem 2009;52:6752-6.

[31] Pantoliano MW, Petrella EC, Kwasnoski JD, Lobanov VS, Myslik J, Graf E, et al. HighDensity Miniaturized Thermal Shift Assays as a General Strategy for Drug Discovery. J Biomol Screen 2001;6:429-40.

[32] Jakubec P, Cockfield DM, Helliwell M, Raftery J, Dixon DJ. Stereoselective, nitroMannich/lactamisation cascades for the direct synthesis of heavily decorated 5-nitropiperidin-2ones and related heterocycles. Beilstein J Org Chem 2012;8:567-78.

[33] Johnson KM, Rattley MS, Sladojevich F, Barber DM, Nuñez MG, Goldys AM, et al. A new family of cinchona-derived bifunctional asymmetric phase-transfer catalysts: Application to the enantio- and diastereoselective nitro-Mannich reaction of amidosulfones. Org Lett 2012;14:2492-5.

[34] Deschout H, Raemdonck K, Demeester J, De Smedt SC, Braeckmans K. FRAP in pharmaceutical research: Practical guidelines and applications in drug delivery. Pharm Res 2014;31:255-70.

[35] Chen P, Chaikuad A, Bamborough P, Bantscheff M, Bountra C, Chung C, et al. Discovery and Characterization of GSK2801, a Selective Chemical Probe for the Bromodomains BAZ2A and BAZ2B. J Med Chem 2016;59:1410-1424.

[36] Hay D, Brennan P, Fedorov O, Tallant C, Monteiro O, Muller-Knapp S, et al. Design and synthesis of potent and selective inhibitors of BRD9 and BRD7 bromodomains. Med Chem Commun 2015;6:1381-6.

[37] Eglen RM, Reisine T, Roby P, Rouleau N, Illy C, Bossé R, et al. The use of AlphaScreen technology in HTS: current status. Curr Chem Genomics 2008;1:2-10.

[38] Martin LJ, Koegl M, Bader G, Cockcroft X-L, Fedorov O, Fiegen D, et al. Structure-based Design of an in vivo Active Selective BRD9 Inhibitor. J Med Chem: ASAP. doi 10.1021/acs.jmedchem.5b01865.

[39] Fedorov O, Lingard H, Wells C, Monteiro OP, Picaud S, Keates T, et al. $[1,2,4]$ Triazolo[4,3-a]phthalazines: Inhibitors of Diverse Bromodomains. J Med Chem 2014;57:462-76.

[40] Picaud S, Strocchia M, Terracciano S, Lauro G, Mendez J, Daniels DL, et al. 9H-Purine Scaffold Reveals Induced-Fit Pocket Plasticity of the BRD9 Bromodomain. J Med Chem 2015;58:2718-36.

[41] Albrecht BK, Cote A, Crawford T, Fauber B, Huang H-R, Lora JM, et al, Genentech, Inc., 
Constellation Pharmaceuticals, Inc. Treating TH2-mediated diseases by inhibition of bromodomains, WO 2014/144721.

[42] Wang L, Pratt JK, McDaniel KF, Dai Y, Fidanze SD, Hasvold L, et al, Abbvie Inc. Bromodomain inhibitors, WO 2013/097601.

[43] Cullen SJ, Ponnappan S, Ponnappan U. Catalytic activity of the proteasome fine-tunes Brg1mediated chromatin remodeling to regulate the expression of inflammatory genes. Mol Immunol 2009;47:600-5.

[44] Schreiber SL. Small molecules: the missing link in the central dogma. Nat Chem Biol 2005;1:64-6.

[45] Weiss WA, Taylor SS, Shokat KM. Recognizing and exploiting differences between RNAi and small-molecule inhibitors. Nat Chem Biol 2007;3:739-44. 


\section{Figures}

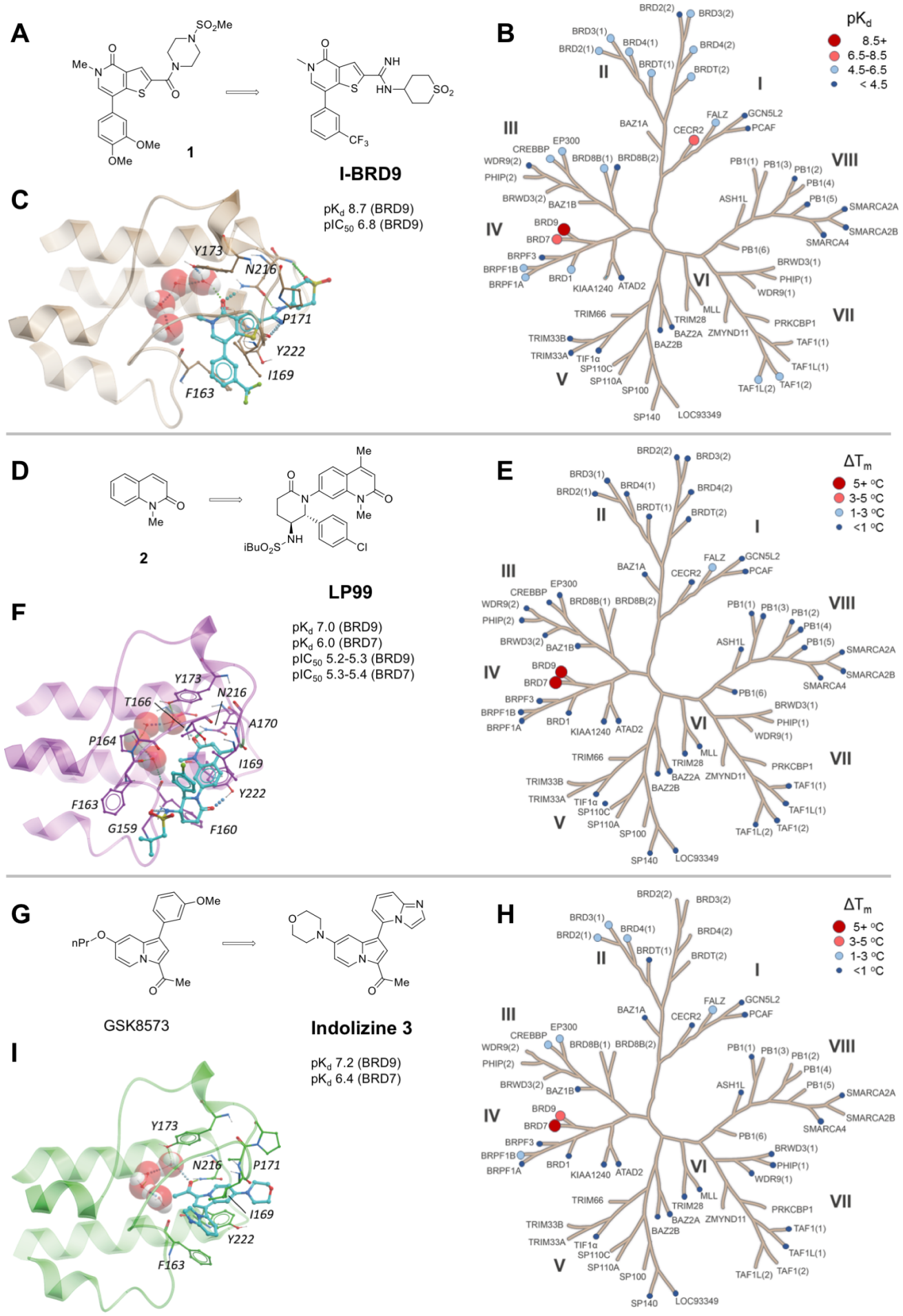

Box 1. Discovery and Characterization of BRD7/9 Bromodomain Probes. A. I-BRD9 and the lead molecule, with affinity assessed by BROMOscan and NanoBRET assays [21]. B. Selectivity screen of I-BRD9 amongst BRD proteins (BROMOscan). C. Co-crystal structure of I-BRD9 with BRD9 (PDB ID 4UIW). D. LP99 and the fragment lead, with affinity assessed by ITC and NanoBRET assays [28]. E. Selectivity screen of LP99 amongst BRD proteins (DSF). F. Co-crystal structure of LP99 with BRD9 (PDB ID 5IGN). G. Indolizine $\mathbf{3}$ and the lead molecule, with the affinity assessed by ITC [35]. H. Selectivity screen of indolizine $\mathbf{3}$ amongst BRD proteins (DSF). I. Co-crystal structure of indolizine 3 with BRD9 (PDB ID 5E9V). 
<smiles>COc1cc(-c2cn([N+](=O)[O-])c(=O)c3cnccc23)cc(OC)c1CN</smiles>

BI-7273<smiles>COc1cc(-c2cn(C)c(=O)c3cnccc23)c(OC)cc1CN</smiles>

BI-9564<smiles>CCOC(=O)Nc1cc(-c2ccc([N+](=O)[O-])c(NS(C)(=O)=O)c2)nn2c(C)nnc12</smiles>

Bromosporine<smiles>O=[N+]([O-])c1nnc2c3ccccc3c(-c3ccc(N4CCOCC4)c(NS(=O)(=O)c4ccccc4Cl)c3)nn12</smiles>

4<smiles>Nc1nc(-c2cc(Br)cc3c2OCC3)c2nc[nH]c2n1</smiles>

5

Figure 1. Other potent BRD7 and BRD9 ligands.
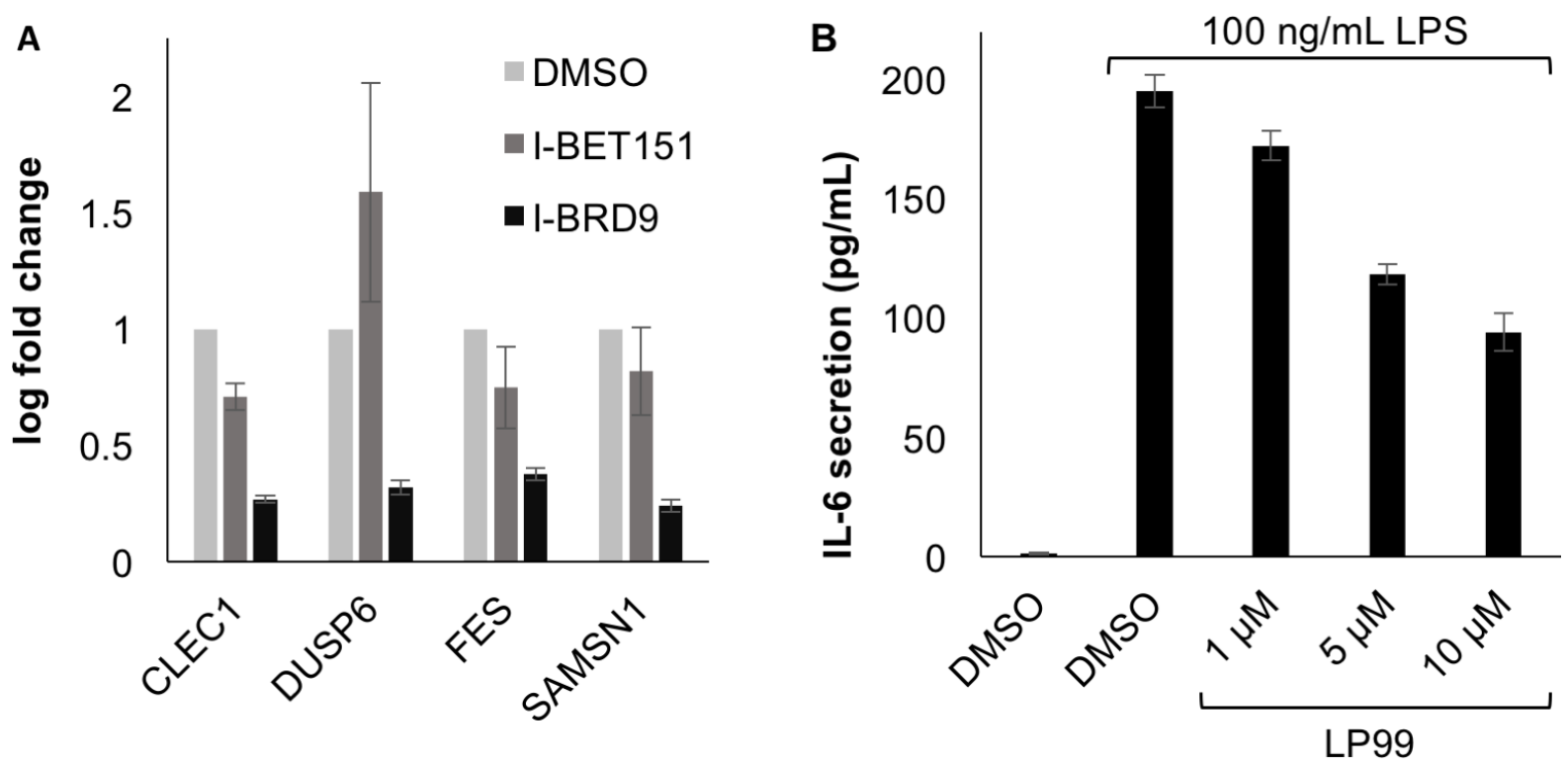

Figure 2. Biological activity. A. Downregulation of selected genes in Kasumi cells by I-BRD9 compared to a BET inhibitor (I-BET151) as assessed by qPCR [21]. B. Inhibition of interleukin-6 secretion (IL-6) from THP-1 cells stimulated with lipopolysaccharide (LPS) [28].

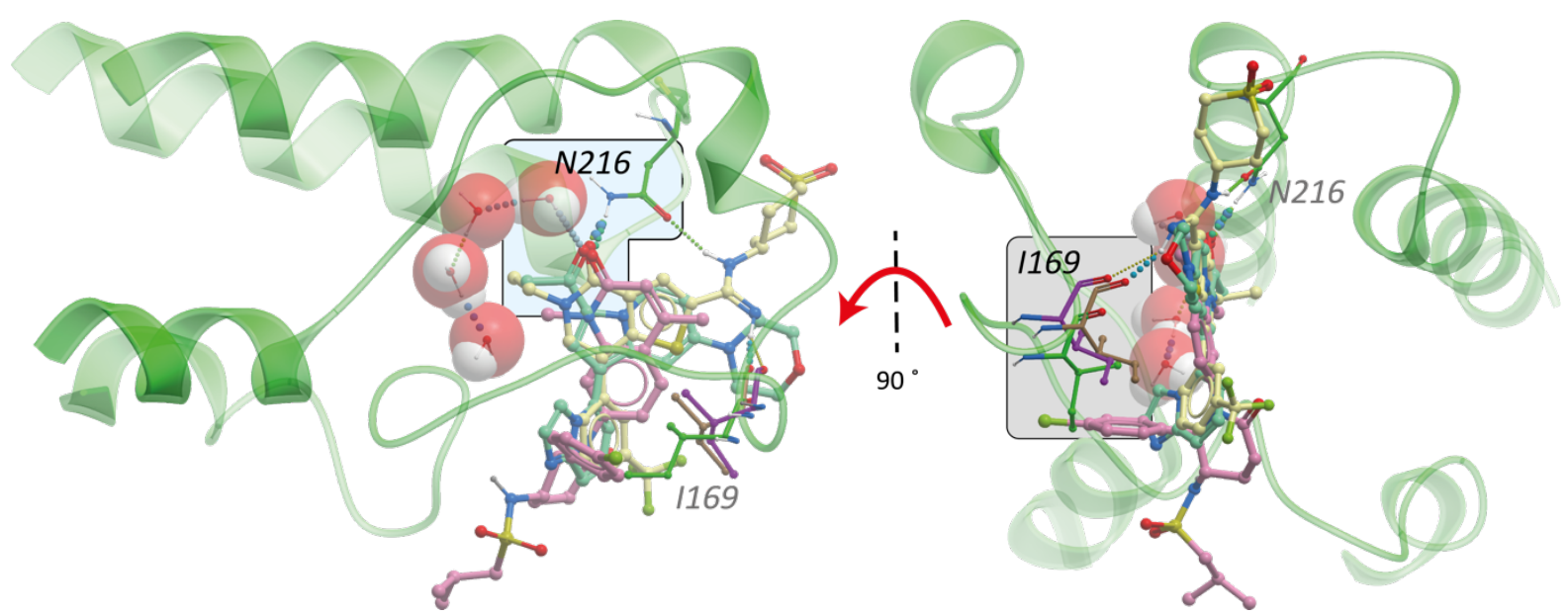

Figure 3. Overlay of I-BRD9 (pale sticks), LP99 (pink sticks), and indolizine 3 (lime sticks) complexes with BRD9 (green ribbon and sticks from complex with 3, PDB ID 5E9V). All ligands 
orient a small alkyl (I-BRD9: Et; LP99, 3: Me) in the pocket and make H-bonds with N216 and the first water molecule in a network of four at the base of the Kac pocket (blue box). The ZA-loop shifts slightly between the structures and I169 is flexible to accommodate the 4-chlorophenyl group of LP99 (grey box). 